\title{
Covid-19: a call for mobilizing geriatric expertise
}

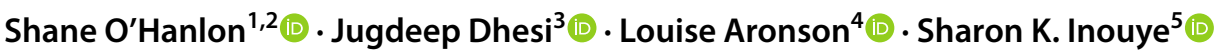

Published online: 23 April 2021

(c) European Geriatric Medicine Society 2021

\section{Key summary points}

Aim Covid-19 is a true gerontological pandemic, with frailty, multimorbidity and geriatric syndromes being of great importance.

Finding There has been a lack of geriatrician involvement in planning and delivery of care for older people with Covid-19 in many arenas. Lack of mobilisation of geriatric expertise has led to inconsistent policy responses and ageism. The pandemic has shown that medical education and health systems have failed to align training, resources, and systems with current demographic and health usage realities.

Messages We must take the specific needs of older people into account to position ourselves to provide better care for this group during Covid-19 and beyond.

Keywords COVID-19 · Frailty · Geriatrics $\cdot$ Ageism $\cdot$ Evidence

\section{Introduction}

The importance of age and frailty in the population affected by Covid-19 has been well described [1], including the disproportionate impact on vulnerable groups such as nursing home residents [2]. Few diseases so epitomise the need for a holistic, age-informed care approach: Covid-19 targets multiple organ systems, preferentially affects older adults with multimorbidity [3], and leads to long-term sequelae including geriatric syndromes of cognitive and functional impairment with the resulting need for rehabilitation [4]. Covid-19 has exposed pervasive ageism in society [5] and negatively affected the mental health of the older adult population, even those without the disease [6]. In short, it is a true gerontological pandemic.

Shane O'Hanlon

shaneohanlon@svhg.ie

St Vincent's University Hospital, Dublin, Ireland

2 University College Dublin, Dublin, Ireland

3 Guy's and St Thomas' NHS Foundation Trust, London, UK

4 University of California, San Francisco, CA, USA

5 Harvard Medical School and Hebrew SeniorLife, Boston, MA, USA

\section{Lack of geriatrician involvement}

For older patients with Covid-19, respiratory, infectious disease or intensive care skills may be necessary but are not sufficient to optimize outcomes. This is true from diagnosis through recovery. The extensive literature on comprehensive geriatric assessment shows it is capable of effectively exploring multiple domains to determine the clinical profile, the pathologic risk as well as the short- and long-term prognosis to facilitate clinical decision-making on the personalized care plan of older persons [7]. Applying this evidence-based model, optimal hospital and after care for Covid-19 in older adults requires general medical skills, shared decision-making, functional and prognostic evaluation, communication of realistic choices, advance care planning, and competence in physical, functional, and cognitive assessment, management and rehabilitation. These skills are well developed in geriatricians, yet they have not been included in the pandemic response in many arenas. Indeed, a significant proportion of older people in hospitals and nursing homes with Covid-19 could be cared for entirely by a geriatrician, or co-managed by a geriatric specialist in areas with limited numbers of geriatricians.

This lack of mobilisation of geriatric expertise has led to inconsistent policy responses, and ageism, with some espousing narratives that misrepresent and devalue older people [8]. Clinical guidelines in some institutions were 
rapidly instigated for children and adults with Covid-19, but the specific needs of older people were generally ignored [9]. Older adults were excluded from most Covid-19 clinical trials, including for vaccines [10], which has limited equitable access to evidence-based treatments and vaccines for the population at highest risk.

\section{Underutilisation of evidence}

Throughout the pandemic, clinicians and health policymakers have underutilised the established geriatric medicine evidence base and missed opportunities to apply proven principles of good geriatric care. It is well established that older people derive benefit from comprehensive geriatric assessment [11]. This is even more relevant in those affected by the pandemic, as geriatric syndromes are highly significant in this group. In Covid-19, frailty has been shown to be associated with earlier death and longer time in hospital, and increasing frailty is associated with increasing rates of mortality [12]. Comorbidity levels are higher in non-survivors compared to survivors, and mortality increases exponentially with each point on the Charlson Comorbidity Index [13]. Dementia is another important factor; in one study, over $75 \%$ of Covid-19 cases in persons with dementia had severe or critical illness on admission and in-hospital mortality was over 60\% [14]. Expertise in frailty, multimorbidity and dementia should be standard of care in Covid-19. However, too much focus on age or multimorbidity risks under-treatment, overtreatment, and the slippery slope of valuing some lives over others; a specific approach for older people, involving appropriate multidisciplinary expertise, is highly relevant [15].

Assessment of the older patient at the 'front door' requires specialist skills. They are more likely to present with 'atypical symptoms' with delirium, asthenia or falls being the main admission complaints in older people, yet not appearing on most Covid-19 symptom lists [16, 17]. Underlying comorbidities mask symptoms, such that older people with Covid-19 reported fewer symptoms than younger groups and this added complexity to diagnosis and treatment [18]. Communication issues have been made more challenging during the pandemic with few health systems recognising the need for transparent masks and hearing or vision aids to support effective communication, prevent delirium, and enable participation in care planning. Restrictions in visiting have had a disproportionate effect on older patients with underlying cognitive impairment. This may have contributed to observed increased prescription of chemical sedation [19]. The under-recognition of the specific needs of older patients remains concerning and the failure to use best practices may have contributed to some of the disproportionate impact of Covid-19 on older patients.

\section{Potential benefits of a geriatric approach during the pandemic}

At presentation, geriatricians can support triage of patients, through applying diagnostic skills in atypical presentation and management of multimorbidity and polypharmacy. Expertise in evidence-based, prognostication-informed, person-centred goals discussions can improve care and ensure resources such as ventilators are appropriately used without rationing [20]. Compassionate, evidence-based, clear communication and shared decisionmaking are vital, since both the Covid-19 infection and its associated treatment can have long-term adverse impacts on quality of life that may be incompatible with a patient's goals. Skills in delivering rehabilitation with a holistic, interdisciplinary approach across medical, functional, social and psychological domains and settings and mobilization of community care resources are also necessary [21]. Rehabilitation should begin on admission to hospital and continue throughout rather than waiting until patients are "medically fit for discharge".

From a public health perspective, the risk of deconditioning in older people is an under-recognized adverse effect from social distancing measures with significant potential to further burden overwhelmed health systems [22]. In healthcare settings, infection control policy restricted many patients to their own room without visitors, but did not adequately acknowledge the risks of reduced mobility or depriving patients from their usual carers. It also lacked integration of a framework to substitute for previous activities such as group exercise programmes. The prevention of deconditioning through physical exercise is a fundamental tenet of geriatric care. Furthermore, the provision of family and paid caregiving, whether funded by the state or privately, was affected during the pandemic and led to difficulty providing for care needs [23]. This has resulted in preventable falls and other conditions that can seriously harm individuals and lead to unnecessary hospitalisation. The absence of geriatrics expertise in public health and policy often meant no one advocated for the hidden workforce of caregivers, without whom many older adults could not remain at home.

\section{Examples of best practice}

Geriatric medicine has a long history of development of novel, evidence-based, cost effective care models. This has continued during the pandemic, as many units established innovative approaches to facilitate urgent assessment. Examples include telephone services for general 
practitioners (GPs) to contact a geriatrician directly for advice, hot clinics (with an appointment available within $24 \mathrm{~h}$ ) and acute frailty units. Delirium prevention programmes with innovative approaches using remote technology have been put in place, including staff training, remote interventions, and delirium prevention toolkits delivered to patients' rooms [24]. Services have also been created that bring timely multidisciplinary care directly to the older person's home and care homes [25], also providing emotional support [26].

We must continue to develop innovative ways to support our nursing homes such as using multiphase emergency response [27], identifying and managing acute illness in the nursing home, facilitating admission when appropriate, and assuring advance care planning with regular updates for all residents [28]. Financing of facilities also needs to be considered in view of inadequate funding and staffing [29]. A series of recommendations has been made for welcoming back visitors [30].

\section{Meeting the new reality of care}

So far during the Covid-19 pandemic, many frail older patients did not receive care from a provider with the geriatric medicine skillset they deserve. Imagine a pandemic in which most patients were children and most clinicians were adult specialists. No one would be surprised by excess preventable suffering and deaths in that context, yet some healthcare professionals and system leaders accept poor care and deaths of older people from Covid-19 as the result of old age, not the failings of our medical care, training, and research. Despite decades of concern about our rapidly ageing population, medical education and health systems have failed to align training, resources, and systems with current demographic and health usage realities. To provide care for older patients equivalent to that already provided to younger adults, we need not only more geriatric specialists but also generalists and specialists with the geriatric knowledge and skills to treat Covid-19 and all other health conditions appropriately in older people.

Looking to the future, we must use this opportunity to further develop our health and social care services not only to align with the needs of the majority of high-risk Covid19 positive population but to generally improve care for the group that is the biggest user of health and social care 'in normal times': older people. We cannot pretend to be doing population health without addressing the needs of this population. We need to navigate and work seamlessly across community and secondary care so that people are able to access quality health care in whatever location optimizes health outcomes and minimizes healthcare costs. In making these changes, we will position ourselves to provide better care to older people during Covid-19 and beyond.

Acknowledgements Dr. Inouye's time is supported in part by Grant R24AG054259 (SKI) from the National Institute on Aging and the Milton and Shirley F. Levy Family Chair at Hebrew SeniorLife/Harvard Medical School.

Funding Dr. Inouye's time is supported in part by Grant R24AG054259 (SKI) from the National Institute on Aging and the Milton and Shirley F. Levy Family Chair at Hebrew SeniorLife/Harvard Medical School.

\section{Declarations}

Conflicts of interest None to declare.

Ethical approval Not applicable.

Informed consent Not applicable.

\section{References}

1. Geriatric Medicine Research C (2021) Age and frailty are independently associated with increased COVID-19 mortality and increased care needs in survivors: results of an international multicentre study. Age Ageing 12:193

2. Miralles O, Sanchez-Rodriguez D, Marco E, Annweiler C, Baz$\tan \mathrm{A}$, Betancor É et al (2021) Unmet needs, health policies, and actions during the COVID-19 pandemic: a report from six European countries. Eur Geriatr Med 12(1):193-204

3. McQueenie R, Foster HME, Jani BD, Katikireddi SV, Sat$\operatorname{tar}$ N, Pell JP et al (2020) Multimorbidity, polypharmacy, and COVID-19 infection within the UK Biobank cohort. PLoS ONE 15(8): $\mathrm{e} 0238091$

4. Karlsson LK, Jakobsen LH, Hollensberg L, Ryg J, Midttun M, Frederiksen $\mathrm{H}$ et al (2020) Clinical presentation and mortality in hospitalized patients aged 80+ years with COVID-19: a retrospective cohort study. Arch Gerontol Geriatr 2020:104335

5. Inouye SK (2021) Creating an anti-ageist healthcare system to improve care for our current and future selves. Nat Aging $1(2): 150-152$

6. De Pue S, Gillebert C, Dierckx E, Vanderhasselt MA, De Raedt R, Van den Bussche E (2021) The impact of the COVID-19 pandemic on wellbeing and cognitive functioning of older adults. Sci Rep 11(1):4636

7. Pilotto A, Cella A, Pilotto A, Daragjati J, Veronese N, Musacchio C et al (2017) Three decades of comprehensive geriatric assessment: evidence coming from different healthcare settings and specific clinical conditions. J Am Med Dir Assoc 18(2):192.e1-e11

8. Savulescu J, Cameron J (2020) Why lockdown of the elderly is not ageist and why levelling down equality is wrong. J Med Ethics 46:717

9. Aronson L. Ageism Is Making the Pandemic Worse. The Atlantic. 2020 March 28.

10. Helfand BKI, Webb M, Gartaganis SL, Fuller L, Kwon C-S, Inouye SK (2020) The exclusion of older persons from vaccine and treatment trials for Coronavirus disease 2019-missing the target. JAMA Intern Med 180(11):1546-1549

11. Parker SG, McCue P, Phelps K, McCleod A, Arora S, Nockels K et al (2017) What is comprehensive geriatric assessment (CGA)? An umbrella review. Age Ageing 47(1):149-155 
12. Hewitt J, Carter B, Vilches-Moraga A, Quinn TJ, Braude P, Verduri A et al (2020) The effect of frailty on survival in patients with COVID-19 (COPE): a multicentre, European, observational cohort study. Lancet Public Health 5(8):e444-e451

13. Iaccarino G, Grassi G, Borghi C, Ferri C, Salvetti M, Volpe M (2020) Age and multimorbidity predict death among COVID-19 patients: results of the SARS-RAS Study of the Italian society of hypertension. Hypertension 76(2):366-372

14. Yao JS, Dee EC, Milazzo C, Jurado J, Paguio JA (2020) Covid-19 in dementia: an insidious pandemic. Age Ageing 49(5):713-715

15. Aronson L (2020) Age, complexity, and crisis: a prescription for progress in pandemic. N Engl J Med 383(1):4-6

16. O'Hanlon S, Inouye SK (2020) Delirium: a missing piece in the COVID-19 pandemic puzzle. Age Ageing 49(4):497-498

17. Clifford CT, Pour TR, Freeman R, Reich DL, Glicksberg BS, Levin MA, et al (2020) Association between COVID-19 diagnosis and presenting chief complaint from New York City triage data. Am J Emerg Med. S0735-6757(20)30986-4. https://doi.org/10. 1016/j.ajem.2020.11.006

18. Unim B, Palmieri L, Lo Noce C, Brusaferro S, Onder G (2021) Prevalence of COVID-19-related symptoms by age group. Aging Clin Exp Res 1:1

19. Howard R, Burns A, Schneider L (2020) Antipsychotic prescribing to people with dementia during COVID-19. Lancet Neurol 19(11):892

20. Ho EP, Neo HY (2021) COVID 19: prioritise autonomy, beneficence and conversations before score-based triage. Age Ageing 50(1):11-15

21. De Biase $S$, Cook L, Skelton DA, Witham M, Ten Hove R (2020) The COVID-19 rehabilitation pandemic. Age Ageing 49(5):696-700

22. Roschel H, Artioli GG, Gualano B (2020) Risk of increased physical inactivity during COVID -19 outbreak in older people: a call for actions. J Am Geriatr Soc 68(6):1126-1128
23. Reckrey JM (2020) COVID-19 confirms it: paid caregivers are essential members of the healthcare team. J Am Geriatr Soc 68(8): $1679-1680$

24. Inouye S. COVID-19 Resources: The Delirium Prevention Toolkit 2020. Available from: https://www.hospitalelderlifeprogram.org/ for-clinicians/covid19-resources/. Accessed 28 March 2021

25. McNamara R, Donnelly K, Boyle N, Menzies D, Hollywood R, Little E et al (2020) Community frailty response service: the ED at your front door. Emerg Med J 37(11):714

26. Lewis SM, Strano-Paul LA (2021) A COVID service-learning initiative: emotional support calls for the geriatric population. $\mathrm{J}$ Am Geriatr Soc 69(2):E4-E5

27. Stall NM, Farquharson C, Fan-Lun C, Wiesenfeld L, Loftus CA, Kain D et al (2020) A hospital partnership with a nursing home experiencing a COVID-19 Outbreak: description of a multiphase emergency response in Toronto, Canada. J Am Geriatr Soc 68(7):1376-1381

28. American Geriatrics Society Policy Brief (2020) COVID-19 and nursing homes. J Am Geriatr Soc 68(5):908-911

29. Lynn J (2020) Playing the cards we are dealt: COVID-19 and nursing homes. J Am Geriatr Soc 68(8):1629-1630

30. Bergman C, Stall NM, Haimowitz D, Aronson L, Lynn J, Steinberg K et al (2020) Recommendations for welcoming back nursing home visitors during the COVID-19 pandemic: results of a delphi panel. J Am Med Dir Assoc 21(12):1759-1766

Publisher's Note Springer Nature remains neutral with regard to jurisdictional claims in published maps and institutional affiliations. 